\title{
Herstellung und Charakterisierung von mittels PLD-Technik gefertigten Bor-dotierten Kohlenstoffelektroden
}

\author{
Manfred Decker ${ }^{1}$, David Haldan ${ }^{2}$, Petra Teichmann ${ }^{2}$, Winfried Vonau ${ }^{2}$, Steffen Weißmantel ${ }^{2}$ \\ ${ }^{1}$ Kurt-Schwabe-Institut für Mess- und Sensortechnik e. V. Meinsberg, Kurt-Schwabe-Str. 4, 04736 \\ Waldheim, Deutschland \\ ${ }^{2}$ Hochschule Mittweida - University of Applied Sciences, Fakultät INW, Technikumplatz 17, 09648 \\ Mittweida, Deutschland \\ decker@ksi-meinsberg.de
}

\begin{abstract}
Zusammenfassung
Mittels Laserpulsabscheidung ist es gelungen, Bor-dotierte Kohlenstoffelektroden auf SiliziumdioxidSubstraten abzuscheiden. Als Haftvermittler kamen $100 \mathrm{~nm}$ dicke Schichten aus Wolframcarbid oder Borcarbid zum Einsatz. Eine $500 \mathrm{~nm}$ Platinschicht diente als leitendes Material zur Kontaktierung der Sensoren. Im nächsten Schritt wurden jeweils Bor-dotierte Kohlenstoffschichten mit einer Dicke von 2 $\mu \mathrm{m}$ abgeschieden. Es wurden Kohlenstofftargets mit variierenden Borgehalten verwendet, um Elektroden mit unterschiedlichen Eigenschaften zu generieren. Zur Verkapselung der Leiterbahnen wurde im abschließenden Prozessschritt Aluminiumoxid mit einer Dicke von $5 \mu \mathrm{m}$ auf die Sensorsubstrate abgeschieden. Um die Möglichkeit einer weiteren Modifizierung der Sensoren aufzuzeigen, wurde erfolgreich die Rippelung der Oberflächen mittels hochenergetischer Laserstrahlung durchgeführt. Dabei konnten gerippelte Profile mit einer Tiefe von $180 \mu \mathrm{m}$ erhalten werden. Elektrochemische Untersuchungen zeigten, dass die elektrochemischen Eigenschaften mit dem Bor-Gehalt der Kohlenstoffschichten der mittels PLD-Verfahren abgeschiedenen Elektroden variieren. Dies bietet interessante Perspektiven für neue elektroanalytische und elektrosynthetische Aufgabenstellungen.
\end{abstract}

Keywords: PLD-Verfahren, Bor-dotierte Kohlenstoff-Elektroden, Elektrolyse, Elektroanalytik, Sensorarray

\section{Zielsetzung}

Die Laserpulsabscheidung (engl. Pulsed Laser Deposition, PLD) bietet interessante Möglichkeiten zur Realisierung von Werkstoffen mit neuen attraktiven Materialeigenschaften. Im Rahmen eines Forschungsvorhabens wird angestrebt, auf planaren Sensorsubstraten mittels des PLD-Verfahrens Kohlenstoffschichten mit verschiedenen Bor-Dotierungen aufzubringen. In elektrochemischen Untersuchungen soll aufgezeigt werden, dass vergleichbar mit Resultaten an Bor-dotierten Diamantelektroden an den mittels PLD generierten Schichten interessante neue analytische und Optionen für die Elektrosynthese erhalten werden können. Die neuartigen Elektrodenmaterialien sollen später in Kombination mit Edelmetallelektroden ein Sensorarray bilden, welches zur elektroanalytischen Bestimmung von redox-aktiven Inhaltsstoffen, wie z.B. Antioxidantien in Lebensmitteln und Getränken dienen kann.

\section{Bor-dotierte Kohlenstoff-Elektroden}

Bor-dotierte Diamant-Elektroden (BDD) stehen im Fokus zahlreicher wissenschaftlicher Arbeiten. Sie zeichnen sich neben ihrer mechanischen und chemischen Stabilität auch durch interessante elektrochemische Eigenschaften aus. Im Fokus der Untersuchungen steht dabei insbesondere die daran erhaltene hohe Überspannung für die Wasserzersetzung, die den Einsatz in wässrigen Medien für viele Anwendungen interessant macht [1, 2]. Darüber hinaus bilden die niedrigen Grundströme die Basis für interessante Forschungsarbeiten, die den Einsatz von Bor-dotierten KohlenstoffElektroden für elektroanalytische Aufgaben im Fokus haben $[3,4]$. Die Herstellung der BDDSchichten erfolgt in der Regel mittels Verfahren der CVD (Chemical Vapor Deposition). Im Rahmen der vorgestellten Untersuchungen soll alternativ die Darstellung von Bor-dotierten Kohlenstoffelektroden mit dem innovativen PLD-Verfahren untersucht werden. 


\section{Grundlagen der Laser-Puls-Abscheidung}

Die Laserpulsabscheidung ist ein Verfahren der physikalischen Gasphasenabscheidung (engl. Physical Vapour Deposition, PVD). Bei dieser Technologie wird der Laserstrahl über das zu verdampfende bzw. abzuscheidende Material (Target) gerastert, wobei die Laserpulsenergie ein schlagartiges Verdampfen des Materials bewirkt. Für dieses Verfahren werden in der Regel Excimer-Laser mit Wellenlängen im UV-Bereich eingesetzt. Die massive Volumenzunahme beim Phasenübergang vom festen in den gasförmigen Aggregatzustand beschleunigt das teilweise ionisierte Material (Plasma) zum gegenüber befindlichen, zu beschichtenden Substrat, auf dessen Oberfläche sich der ablatierte Teilchenstrom als dünne Schicht anlagert. Der prinzipielle Ablauf der PLD ist in Abbildung 1 dargestellt. Dieser Prozess findet im Hochvakuum statt, um eine nahezu ungehinderte Ausbreitung des Targetmaterials zu ermöglichen. Mit dieser Methode lassen sich zahlreiche Materialen ablatieren und auf Substraten abscheiden. Durch die Variation der Laserfluenzen können darüber hinaus auch die Bindungsverhältnisse in den abgeschiedenen Schichten (z.B.: Verhältnis des $\mathrm{sp}^{2}-\mathrm{zu} \mathrm{sp}^{3}$-hybridisiertem Kohlenstoff) beeinflussen. Das Verfahren ermöglicht auch einen exakten stöchiometrischen Übertrag zusammengesetzter Targets sowie eine hohe Packungsdichte der aufwachsenden Schichten. Durch eine gezielte Gaszufuhr bzw. den Einbau von Fremdatomen lassen sich die Eigenschaften der abgeschiedenen Schichten weiter verändern. $[5,6,7]$

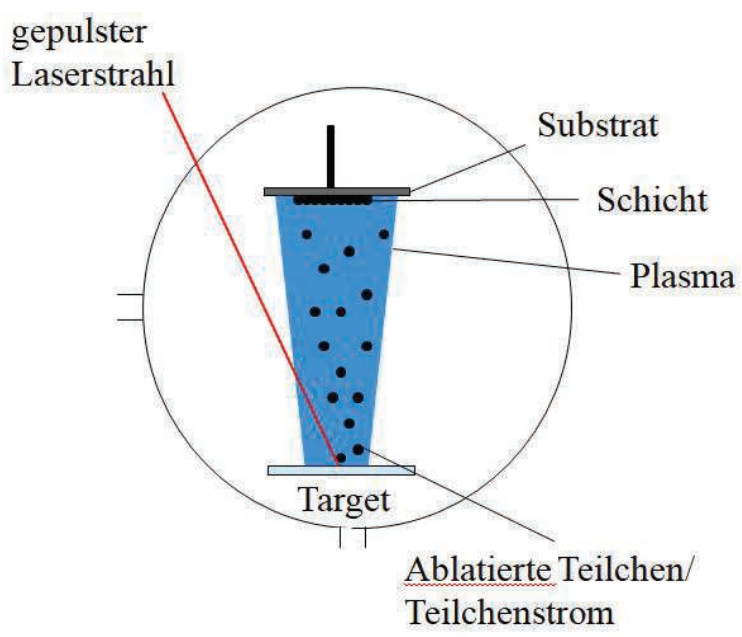

Abb. 1: schematische Darstellung des PLDVerfahrens

\section{Aufbau und Layout eines Sensors}

Zur Herstellung der Sensorstrukturen kamen rechteckige, mit Laser geschnittene Siliziumoxid-Substrate mit den Abmaßen $10 \mathrm{~mm} \times 25$ $\mathrm{mm}$ zum Einsatz. Auf Grund der schlechten Haftung von Edelmetallen und Bor-dotierter Kohlenstoffschichten, bei denen der Kohlenstoff- den Boranteil überwiegt, auf keramischen Substraten, musste ein Haftvermittler aufgebracht werden. Dazu fanden Wolframcarbid und Borcarbid Verwendung. Eine Haftschichtdicke von $100 \mathrm{~nm}$ ist für die Zwecke ausreichend. Die für die Analytik vorgesehene elektrochemische Untersuchung der Sensoren erforderte die Aufbringung einer elektrisch leitenden Schicht zur Kontaktierung der Elektroden. Dazu wurde Platin mit einer Schichtdicke von $500 \mathrm{~nm}$ als Leiterbahn abgeschieden. Auf diese Kontaktschicht folgte die eigentliche Sensorschicht, bestehend aus Bordotiertem Kohlenstoff variierender Zusammensetzung. Auf Grund großer Partikulate in den Schichten, welche vor allem durch Bor gebildet werden, musste eine ausreichende Schichtdicke realisiert werden, um die Dichtheit der Sensorelektrode sicherzustellen. Dies dient zur Abdeckung der Haft- sowie der Kontaktschicht vor dem zu analysierenden Medium, um eine Verfälschung der Messungen durch störende Umsetzungen an diesen Materialien auszuschließen. Eine Schichtdicke von $2 \mu \mathrm{m}$ erwies sich als ausreichend. Die Isolierung der Sensorbahnen erfolgte durch eine abschließende Schutzschicht. Als geeignetes Material dafür wurde Aluminiumoxid ausgewählt, welches eine sehr hohe Dichtheit aufweist und als inerte Keramik keine chemische Reaktionen mit den zu analysierenden Substanzen zeigt. Weiterhin lässt sich das Material sehr gut mittels PLD-Verfahren ablatieren und hat eine vergleichsweise hohe Aufwachsrate. Bei der Abscheidung wurde eine Schichtdicke von 5 $\mu \mathrm{m}$ zum Schutz der darunterliegenden Leiterbahn gewählt. Abbildung 2 zeigt den Aufbau.

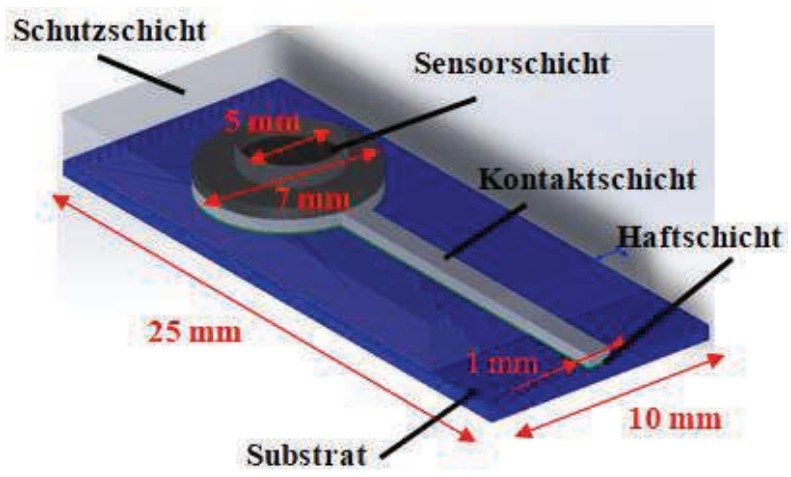

Abb. 2: Sensoraufbau und -Layout 


\section{Versuchsaufbau und -durchführung}

Die Herstellung der Sensoren erfolgte unter Vakuum bei Drücken $<10^{-5}$ mbar in einem Rezipienten. Für die Ablation der Materialien kam ein Ecximer-Laser mit einer Laserwellenlänge von $248 \mathrm{~nm}$ und einer Pulsenergie von $770 \mathrm{~mJ}$ zum Einsatz. Die Strahlablenkung des Laserstrahls für die Ablatierung der Targets erfolgte mittels einer Ablenkeinheit. Dadurch war eine spiralförmige Abrasterung des Targets durch den Laserstrahl möglich. Diese Bewegung und eine zusätzliche Rotation des Substrates hat eine bessere Homogenität der Schichtverteilung auf dem Substrat zu Folge. Die Reinigung der Substrate erfolgte durch eine lonenstrahlreinigung mittels einer Kaufmannionenquelle. Hohe Eigenspannungen von bis zu $10 \mathrm{GPa}$ in den aufwachsenden Sensorschichten erforderten eine Entspannung der einzelnen Schichtlagen. Dazu muss ein zweiter Excimer-Laser gleicher Laserwellenlänge eingesetzt werden. Der Unterschied der beiden Excimer-Laser liegt ausschließlich im Aufbau des Resonators. Für die Ablation ist es von Vorteil, einen instabilen Resonator zu verwenden, während man bei der Entspannung der Schichten einen stabilen Resonator benutzt. Während der Ablationslaser auf das Target fokussiert ist, wird der Entspannungslaser auf die aufwachsenden Schichten gelenkt. Eine weitere Ablenkeinheit, verknüpft mit dem Entspannungslaser, sorgt für ein mäanderförmiges Abrastern des Substrates. Dadurch lassen sich die Schichteigenspannungen auf unter 2 GPa reduzieren. Abbildung 3 zeigt den Aufbau.

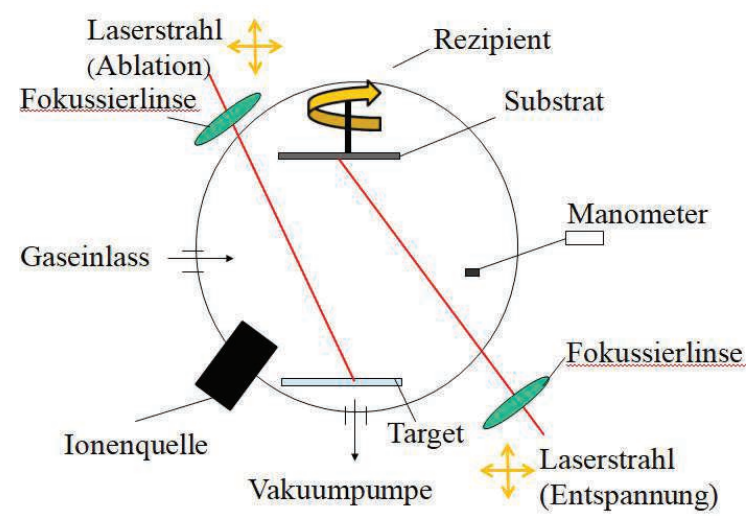

Abb. 3: Versuchsaufbau mit Ablations- und
Entspannungslaser

Durch Verwendung einer aus einem dünnen Edelstahlblech zugeschnittenen Maske ließ sich das in Abb. 2 gezeigte Layout des Sensors auf dem Substrat erzeugen. Die Maske wird dazu auf dem Substrat fixiert. Vor jeder Beschichtung erfolgte über eine Minute eine lonenstrahlreinigung des Substrats. Im Anschluss daran begann das Abscheiden der 100 $\mathrm{nm}$ dicken Haftschicht bei einer Laserfluenz von $7-9 \mathrm{~J} / \mathrm{cm}^{2}$. Die drauf folgende $500 \mathrm{~nm}$ dicke Kontaktschicht aus Platin wurde bei einer Laserfluenz von ca. $7 \mathrm{~J} / \mathrm{cm}^{2}$ ablatiert. Nach anschließender Modifikation der Maske in Form eines Kreises mit einem Durchmesser von sieben Millimetern erfolgte die Erzeugung der Sensorschicht aus einem Bor-dotiertem Kohlenstofftarget. Dabei wurde die Herstellung in mehrere Schritte unterteilt. Zuerst wurde eine $100 \mathrm{~nm}$ dicke Sensorschicht aufgebracht und diese im Nachgang mit dem Entspannungslaser behandelt. Diese Vorgehensweise erfolgte so lange, bis eine Gesamtschichtdicke von ca. $2 \mu \mathrm{m}$ erreicht war. Neben Borcabid $\left(\mathrm{B}_{4} \mathrm{C}\right)$ wurden unter anderem noch die Bordortierten Kohlenstoffe mit 10\% ( $\left.B_{10} C_{90}\right), 25 \%$ $\left(\mathrm{B}_{25} \mathrm{C}_{75}\right)$ und $50 \%\left(\mathrm{~B}_{50} \mathrm{C}_{50}\right)$ Boranteil als Target verwendet. Die Abscheidung erfolgte bei unterschiedlichen Laserfluenzen. Dadurch war es möglich, den $\mathrm{sp}^{3}$ - und $\mathrm{sp}^{2}$-hybridisiertem Anteil des Kohlenstoffs zu variieren. Mit steigender Laserfluenz nimmt der $\mathrm{sp}^{3}$-hybridisierte Kohlenstoffanteil asymptotisch zu. Jedes der vier Bor-dotierten Kohlenstofftargets wurde jeweils bei den Laserfluenzen 5, 7, 9 und $11 \mathrm{~J} / \mathrm{cm}^{2}$ ablatiert. Nach Fertigstellung der Sensorschicht und dem Austausch der Maske erfolgte das Aufbringen der $5 \mu \mathrm{m}$ dicken Schutzschicht aus Aluminiumoxid bei einer Laserfluenz von ca. $7 \mathrm{~J} / \mathrm{cm}^{2}$. Alle erzeugten Schichten sind von amorpher Struktur.
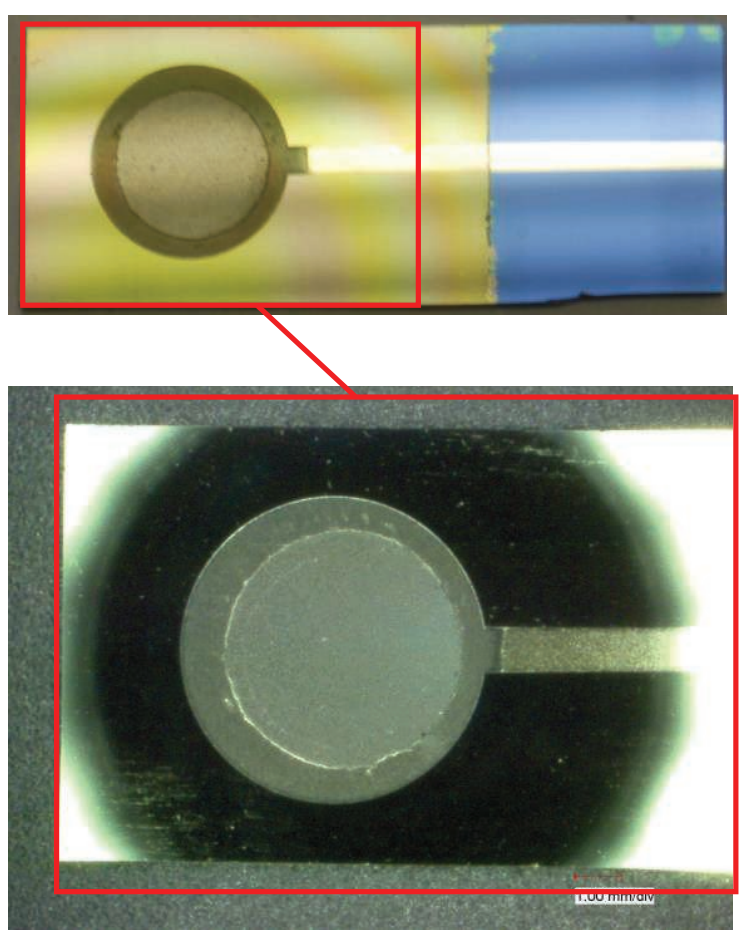

Abb. 4: oben: Lichtmikroskopische Aufnahme der Sensorfläche, unten: 20fache Vergrößerung unter Lichtmikroskop 
Die Sensorschichten wiesen eine Rauheit von 5-250 nm auf. Diese ist stark von der Laserfluenz und vom Borgehalt abhängig. Vor allem Schichten mit hohem Borgehalt $\left(\mathrm{B}_{4} \mathrm{C}\right)$ weisen gegenüber den anderen Schichten eine sehr hohe Rauheit auf.

\section{Modifikation der Sensorschicht}

Eine Möglichkeit der Modifikation der Sensorschichten bietet das sogenannte Rippeln. Gerippelte Strukturen sind in Folge von Laserstrahlung entstandene selbstorganisierende, periodische Oberflächenstrukturen. Sie sind abhängig vom einfallenden Winkel des Laserstrahls und dessen Polarisationsrichtung. Diese periodische wellenförmige Struktur entsteht nahe der Ablationsschwelle des Materials $[8,9,10]$.

Die Rippel sollen vor allem zu einer Vergrößerung der Kontaktfläche und damit zu einer Erhöhung der Sensitivität der Sensoren führen. Für das Rippeln der Sensorfläche kam ein Femtosekunden-Laser mit einer Wellenlänge von 1028 nm zum Einsatz. Die Probe wurde mit einem Positionierungssystem unter einen stationären Laserstrahl bewegt, der einen Strahlradius von $11,5 \mu \mathrm{m}$ im Fokus und eine Pulsrepetitionsrate von $200 \mathrm{kHz}$ sowie eine Pulsdauer von ca. 220 fs aufwies. Durch die hohe Anzahl an Partikulaten in der Sensorschicht war für eine zuverlässige Rippelbildung eine doppelte Überfahrt notwendig.

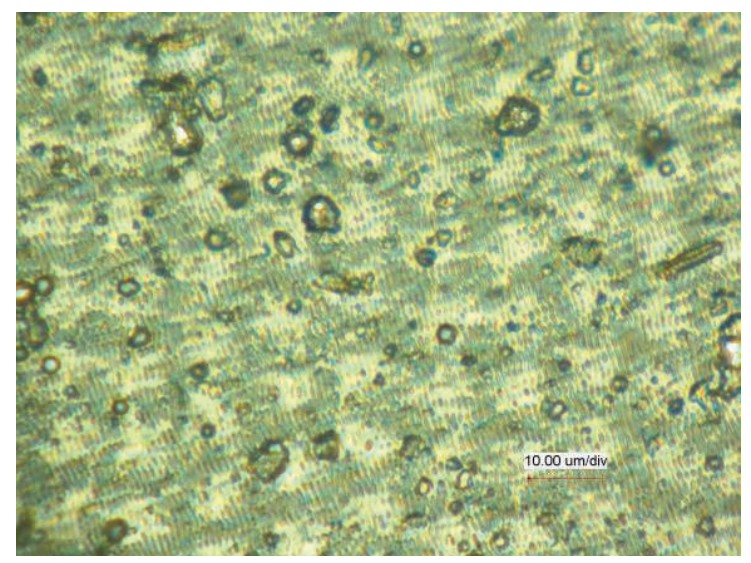

Abb. 5: lichtmikroskopische Aufnahme der gerippelten Sensorfläche, 3000fach vergrößert

Die Abbildungen 5 bis 7 zeigen mikroskopische Aufnahmen der gerippelten Sensoroberflächen und das dazugehörige Profil.

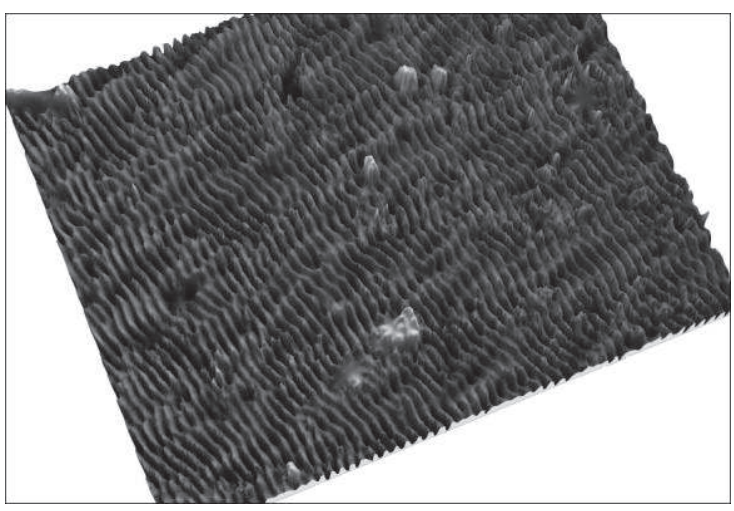

Abb. 6: lasermikroskopische Aufnahme der gerippelte Sensorfläche (Objektiv 50fache Vergrößerung)

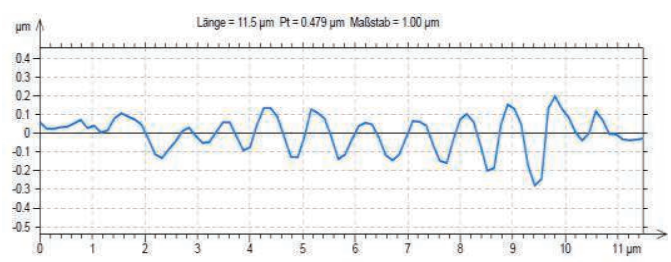

Abb. 7: Profil einer 11,5 $\mu \mathrm{m}$ langen gerippelten Strecke

Auf der Sensorfläche wurde eine Fläche von ca. $16 \mathrm{~mm}^{2}$ gerippelt. Die Rippel wiesen eine Durchschnittstiefe von ca. $180 \mathrm{~nm}$ auf und einen Abstand von $900 \mathrm{~nm}$ voneinander.

\section{Verhalten im Elektrolyten}

Elektrochemische Messungen zeigten, dass mittels PLD-Verfahren abgeschiedene Bordotierte Kohlenstoffmaterialien in einem Puffer $\mathrm{pH}$ 4,01 mit einem $10^{-2}$ molarem $\mathrm{KCl}$-Gehalt ausgeprägte Stromspannungskurven aufweisen (Abbildung 8). Die kathodischen Ströme waren, im Vergleich zu reinem Platinblech, deutlich geringer ausgeprägt bei den Elektroden basierend auf Borcarbid $\left(\mathrm{B}_{4} \mathrm{C}\right)$ bzw. bei der Kohlenstoffelektrode mit $50 \%$ Borgehalt (B50C50). Besonders auffällig ist der anodische Anstieg des Stromes ab einem Potenzial von $+0,6 \mathrm{~V}$ vs. SSE bei der PLD-Schicht, die aus reinem Borcarbid gebildet wurde. 


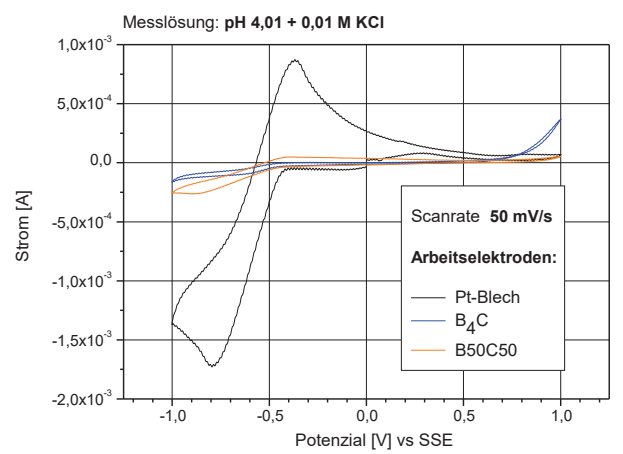

Abb. 8: Stromspannungskurve von zwei Bordotierten Kohlenstoffelektroden im Vergleich zu einer Pt-Elektrode in Puffer pH 4,01 mit 0,01 M Gehalt an $\mathrm{KCl}$ (Elektrodenfläche alle 20,0 $\mathrm{mm}^{2}$ )

Um die Eignung der mittels PLD-Verfahren hergestellten Elektroden auch für elektroanalytische Messungen aufzuzeigen, wurden cyclovoltammetrische Untersuchungen mit gelben Blutlaugensalz in $0,01 \mathrm{M} \mathrm{KCl}$ durchgeführt. In Abb. 9 ist zu erkennen, dass an Kohlenstoffelektroden mit einem 50\%igem Gehalt an Bor keine Redoxreaktion beobachtet wird, während ein 10\%iger-Gehalt deutlich die reversible Reaktion des gelben Blutlaugensalzes zeigt. Die Höhe der Ströme betrug $40 \%$ des Wertes an einer nicht dargestellten Platin-Elektrode gleicher Fläche. Auch hier zeigt sich bei der Elektrode, die aus einem $\mathrm{B}_{4} \mathrm{C}$-Target hergestellt wurde, ein deutlicher Stromanstieg im anodischen Bereich, dem in diesem Elektrolyten bei $+0,6 \mathrm{~V}$ vs. SSE noch ein irreversibler Oxidationspeak vorausgeht. Allen Elektroden ist nur ein geringer kathodischer Strom bis $-1,0$ $V$ vs. SSE gemeinsam. Dies legt die Vermutung nahe, dass nur eine kleine Platinfläche der Ableitschicht auf dem Substrat mit der Messlösung in Kontakt steht.

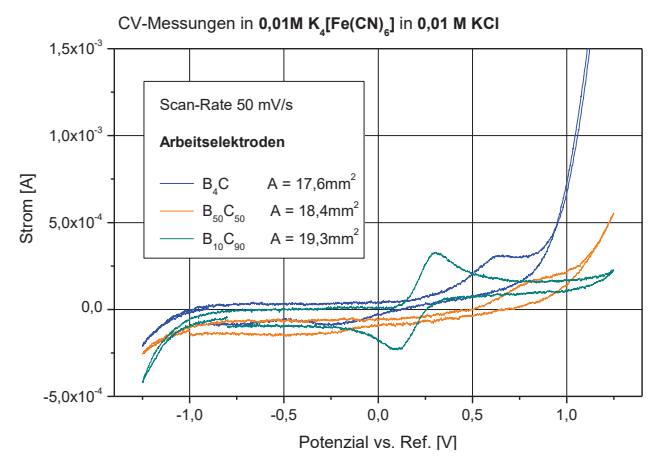

Abb. 9: Cyclovoltammogramm von drei Bordotierten Kohlenstoffelektroden in $0,01 \mathrm{M} \mathrm{KCl}$ mit 0,01 $\mathrm{M}$ Gehalt an $\mathrm{K}_{4}\left[\mathrm{Fe}(\mathrm{CN})_{6}\right]$
In zukünftigen Untersuchungen sollen die Elektrodenstrukturen mit den mittels PLD-Verfahren realisierten Bor-dotierten Kohlenstoffschichten in weiteren Pufferlösungen und mit verschiedenen, anorganischen und organischen Redox-Systemen tiefergehender auf ihre elektrochemischen Eigenschaften charakterisiert werden.

\section{Ausblick}

Die ersten elektroanalytischen Messungen zeigen für verschiedene, mittels PLD-Verfahren gefertigte Kohlenstoffelektroden mit variierendem Bor-Gehalt unterschiedliches Verhalten in verschiedenen elektrolytischen Lösungen. Diese Resultate legen nahe, dass durch die Laserpulsabscheidung neue Materialien mit interessanten elektrochemischen Eigenschaften generiert werden können. In vertiefenden Messreihen werden weitere Elektrolyte und elektroaktive Substanzen an den neuen innovativen Elektrodenmaterialien untersucht werden. Darüber hinaus wird eine nähere Charakterisierung der Kohlenstoffschichten mit physikochemischen Methoden erfolgen.

\section{Danksagung}

Die Forschungsarbeiten werden freundlicherweise vom BMBF über das Programm KMUinnovativ unter den Förderkennzeichen 031B0182C und 031B0182D unterstützt.

[1] E. Brillas, C.A. Martinez-Huitle, Synthetic Diamond Films. Wiley-Verlag, Hoboken (NJ) (2011) 79-108 und 155-180

[2] R. L. McCreery, Chemical Reviews 108, 2646-2687; doi: 10.1021/cr068076m

[3] V. A. Pedrosa, A. R. Malagutti, L. H. Mazo, L.A. Avaca, Analytical Letters 39, 27372748 (2006); doi: http://dx.doi.org/10.1080/00032710600867 317

[4] O. Chailapakul, E. Popa, H. Tai, B. V. Sarada, D. A. Tryk, A. Fujishima, Electrochemical Communications 2, 422-426 (2000); https://doi.org/10.1016/S13882481(00)00049-7

[5] G. Kienel, Vakuumbeschichtung. SpringerVerlag, Berlin (1997) 80-84

[6] B. Dischler, C. Wild, Low-Pressure Synthetic Diamond - Manufacturing and Aplications. Springer-Verlag, Berlin (1998)

[7] D. Bäuerle, Laser Processing and Chemistry. Springer-Verlag, Berlin, Heidelberg (1996)

[8] J. E. Sipe, J. F. Young, J. S. Preston, H. M. v. Driel, Physical Review B 27, 1141-1154 
(1983); doi:

https://doi.org/10.1103/PhysRevB.27.1141

[9] P. M. Fauchet, A. E. Siegman, Applied

Physics Letters 40, 824-826 (1982); doi: https://doi.org/10.1063/1.93274

[11] J. E. Sipe, J. F. Young, J. S. Preston, H. M. v. Driel, Physical Review B 27 (1983)

1155-1172 (1983); doi:

https://doi.org/10.1103/PhysRevB.27.1155 